\title{
Comparability the geometric resolution of IR thermograph defined in various ways
}

L. Rozanski*

*Division of Metrology and Measurement Systems, Institute of Mechanical Technology, Poznan University of Technology, pl. M. Sklodowskiej-Curie 5, 60-965 Poznan, Poland, leszek.rozanski@gmail.com

\section{Introduction}

The geometrical (spatial) resolution is defined as the thermograph ability to reproduce the geometrical details in the thermal image. There are many ways in literature to define the geometric resolution of the thermal imaging systems [1], [2], [3], [4]. Sometimes makes it difficult to compare the metrological features of of these devices carried to determine their ability to distinguish in the thermal images of tested objects their geometric details. The paper presents some of the most common ways to define this parameter and the method of comparing their values.

\section{Methods of defining the geometric resolution of IR thermograph}

In technical and scientific articles are used a number of different ways of defining geometric resolution of the thermographs. Here are a few of these definitions.1) The geometric resolution is determined by the angular size of the active detector area (pixel) transferred to the object space of the camera optical system (GR1). 2) The geometric resolution is defined as the angle at which the camera "sees" a single flat object with the width of the vertical at which the maximum value of the measurement signal is $50 \%$ of the signal corresponding to the temperature of the large object (GR2) [3], fig. 1 and fig 2. It is assumed that all points of this object have the same temperature. Using the function of SRF (Slit Response Function) GR2 can be defined as such an argument value of SFR for which a value to this function is 0.5 .

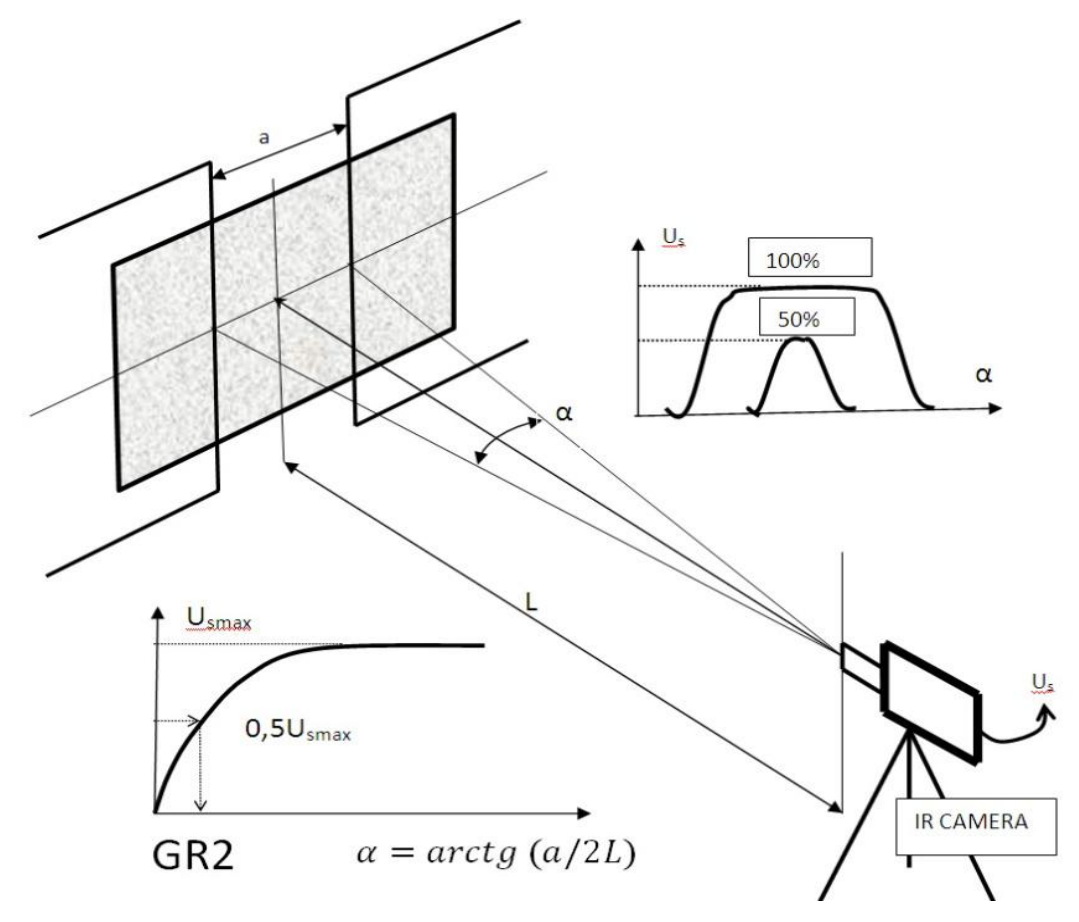

Fig. 1 Methods of measuring the geometrical resolution according to 2 definition 


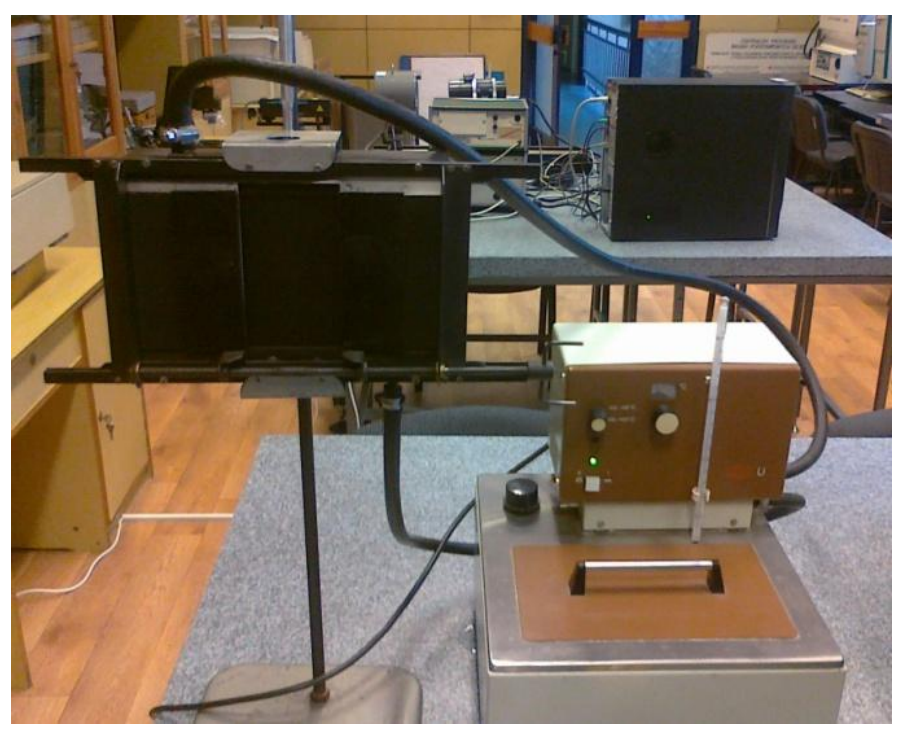

Fig. 2 Apparatus for the measurement of the geometrical resolutions according to 2 definition

3) The geometric resolution is determined as that angular width at which the line spread function (LSF) of the thermograph has dropped to $40 \%$ (GR3) [2], fig. 3 and fig 4. 4) The geometric resolution is determined as that half the inverse of spatial frequency at which the modulation transfer function (MTF) of the thermograph has dropped to $50 \%$ (GR4) [1].

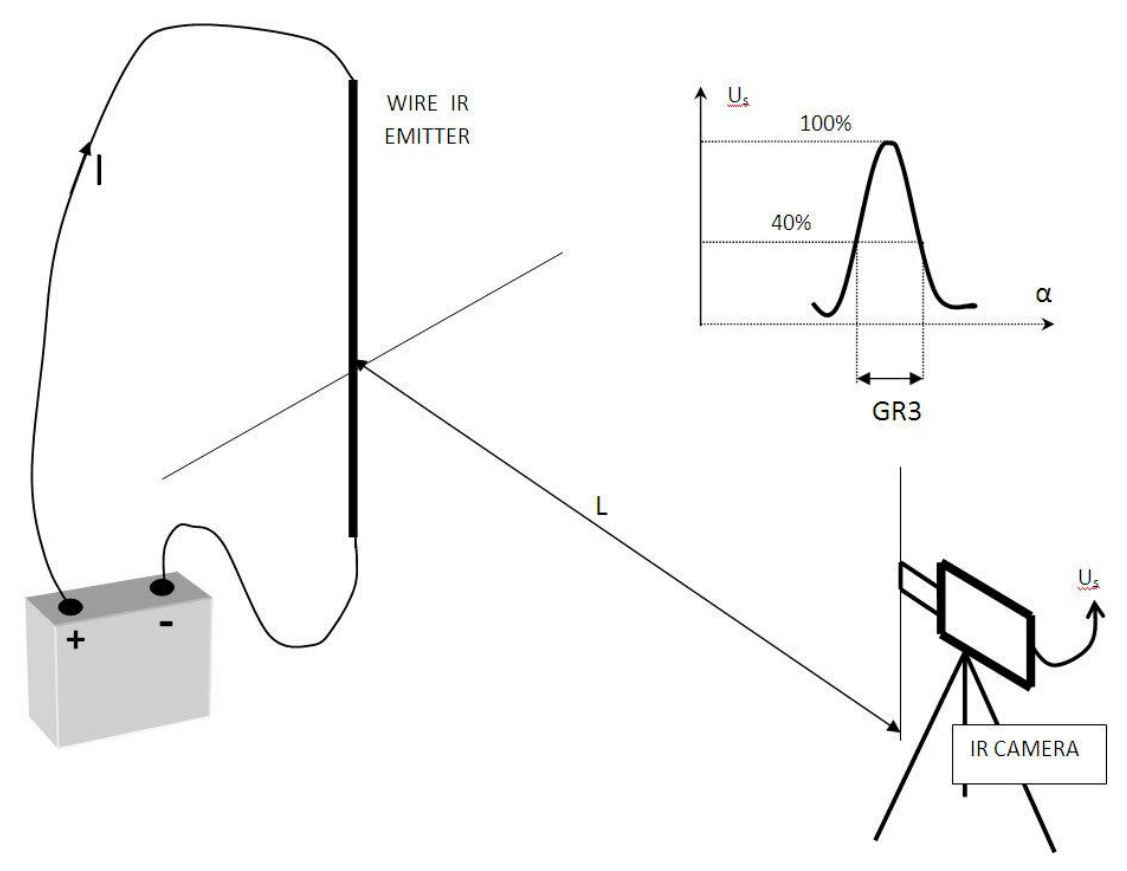

Fig. 3 Methods of measuring the geometrical resolution according to 3 definition

\section{Comparison of geometric resolution defined by various methods}

It is easy to see that the definition of 1 can not be compared with the definitions 2-4. This is because it combines the geometric resolution of the thermograph only with the parameters describing the geometry of the optical system. It does not take into account in this case, the impact of optical aberrations, detector time constant and filtration of the electronic circuit. Comparative analysis of definition of 2-4 will be referenced to a class thermographic 
devices that filter characteristics can be described using the theory of linear filtering. Based on literature data it was assumed that, for thermograph properly designed and constructed, the LSF function can be approximated by a Gaussian function:

$$
\operatorname{LSF}(\alpha)=\exp \left(-\frac{\alpha^{2}}{\sigma^{2}}\right)
$$

where: $\quad \alpha$ - angular coordinate, $\sigma-$ a constant value, expressed in angular units.



Fig. 4 The wire IR emitter with the pulse temperature controller

According to the definition 2, geometric resolution GR2 of the thermograph can be determined by calculating the value of the angle $\alpha_{o}$ for which the equation (2) is fulfilled:

$$
\frac{\int_{-\frac{\alpha_{o}}{2}}^{\frac{\alpha_{o}}{2}} \operatorname{LSF}(\alpha) d \alpha}{\int_{-\infty}^{+\infty} \operatorname{LSF}(\alpha) d \alpha}=\frac{1}{2}
$$

where: $\alpha_{0}$ - angular coordinate value.

Thus, in accordance with the definition $2, G R 2=\alpha_{0}$. According to the definition 4, geometric resolution GR4 resolution can be obtained by determination of such a value of spatial frequency $f_{p a}$ defined in the image plane, for which the equation (3) is fulfilled:

$$
\operatorname{MTF}\left(f_{p \alpha}\right)=1 / 2
$$

Thus:

$$
G R 4=1 / 2 f_{p \alpha}
$$

Taking into account that:

$$
\operatorname{MFT}\left(f_{p \alpha}\right)=\frac{\left|\int_{-\infty}^{+\infty} \operatorname{LSF}(\alpha) e^{-j 2 \pi \alpha f_{p \alpha}} d \alpha\right|}{\left|\int_{-\infty}^{+\infty} \operatorname{LSF}(\alpha) d \alpha\right|}
$$

as well as considering that LSF function can be approximated by a Gaussian function, then from formulas (1) and (5) we obtain:

$$
\operatorname{MTF}\left(f_{p \alpha}\right)=\exp \left(-\pi^{2} \sigma^{2} f_{p \alpha}^{2}\right) .
$$


After taking into account relations (3) and (6) we get:

$$
G R 4=\frac{\pi \sigma}{2 \sqrt{\ln 2}} \approx 1.88 \sigma
$$

In a similar manner as above can be shown that:

$$
G R 3=2 \sqrt{\ln 10 / 4} \sigma \approx 1.91 \sigma
$$

and that

$$
G R 2 \approx 0.96 \sigma .
$$

\section{Summary}

It is possible to convert the geometric resolution values defined in various ways. It is not possible to do this only in the case of the definition, which combines the geometric resolution of the thermograph only with the parameters describing the geometry of the optical system.

\section{REFERENCES}

[1] Poloszyk S. Rozanski L. , "Influence of the radiation diffraction in image converter of the thermograph upon its metrological parameters”. Proceedings of the QIRT'96, pp. 20-25, Stuttgart,1996.

[2] Vermeij G. F., Eland M., Steketee L., "MTF measurement, a simple method for determining the spatial resolution of a thermograph". Acta Thermographica, pp.143-147, 1980.

[3] Rudowski G., "Termowizja I jej zastosowania”. WKiŁ, Warszawa, 1979.

[4] Maldague X. P. V.,"Theory and Practice of Infrared Technology for Nondestructive Testing" JOHN WILEY \& SONS INC., 2001. 